\title{
Mass flow rate of fine and cohesive powders under differential air pressure
}

\author{
R. Baserinia ${ }^{1,2}$ and I.C. Sinka ${ }^{2 *}$ \\ 1. School of Engineering and Sustainable Development, Faculty of Technology, De Montfort \\ University, UK \\ 2. Department of Engineering, University of Leicester, UK \\ * Corresponding author ics4@le.ac.uk \\ Revised manuscript to Powder Technology \\ 24 March 2018
}

\begin{abstract}
Air-powder interactions are of practical importance in the production of pharmaceuticals, food and high value added chemicals manufactured using powders. For examples, air-powder effects enable consistent and effective dosing of fine cohesive powders into dies on high productivity rotary presses due to the suction fill effect.

A purpose built experimental testing rig was developed and calibrated in order to develop a basic understanding of effect of air pressure on the mass flow rate of fine and cohesive powders. The powder materials were selected to enable the study of the effect of particle properties, such as size and density, and processing conditions such as differential air pressure, on the mass flow rate of powders.

The models available in the literature developed for coarse free flowing sands under differential pressure were found inadequate to describe the experimental observations and to predict the flow behaviour of fine and cohesive powders. A new powder flow model was developed using established dimensional analysis methods based on the experimental data. The proposed model includes terms that account for the effect of differential pressure and reduces to the classic Beverloo model in absence of differential pressure. The models was validated and can be used for formulation and process design for flow regimes where air-powder interactions are important.
\end{abstract}

\section{Keywords}

Powder flow, fine granular solids, bin discharge, air pressure gradient, discharge rate 


\section{Notations}

$\dot{m}_{B} \quad$ Mass flow rate predicted by Beverloo correlation

$\dot{m}_{p} \quad$ Additional mass flow rate induced by differential pressure

$\Delta P_{0} \quad$ Adverse pressure gradient to prevent flow

$\Phi_{p} \quad$ Particle-air density ratio

$A_{c} \quad$ Average Cross section area of container

$A_{o} \quad$ Cross section area of orifice

$D_{c} \quad$ Average diameter of container

$b_{0} \quad$ Width of the orifice

$f f_{c} \quad$ Critical flow factor

$\dot{m} \quad$ Mass flow rate

$r_{0} \quad$ Radius of free fall arch

$\beta_{1} \quad$ Parameter in Equation 8

$\beta_{2} \quad$ Parameter in Equation 8

$\mu_{p} \quad$ Coefficient of friction between particles

$\mu_{p w} \quad$ Coefficient of friction between particles and wall

$\rho_{b} \quad$ Bulk density

$\Delta P \quad$ Differential pressure

$A \quad$ Cross section area of powder bed

Ar Archimedes number

$B \quad$ Parameter in Equation 9

$D \quad$ Orifice diameter

$H \quad$ Height of powder in the container

$K \quad$ Powder permeability

$L \quad$ Length of powder bed

$Q \quad$ Volumetric flow rate of fluid

$Z \quad$ Correction factor in Weighardt relation

a Empirical constant

c Empirical constant

$d \quad$ Averaged particle size

ff Flow factor

$g \quad$ Acceleration due to gravity

$k \quad$ Dimensionless constant related to the shape of particle

$l \quad$ Hopper thickness

$n \quad$ Empirical constant

$\alpha \quad$ Half angle of the hopper

$\varepsilon \quad$ Powder voidage

$\lambda \quad$ Empirical value dependent on compressibility and gas properties

$\mu \quad$ Viscosity of fluid 


\section{Introduction}

Hoppers and bins are used in many industry sectors involving the transport and storage of granular materials. Large-scale applications include mineral extraction and processing, construction, bulk shipment of agricultural products etc. Fine and cohesive powders are present mostly at small scale handling and dosing processes such as filling of pharmaceutical capsules, food, and fine chemicals typically used in high value-added products. In these operations, the flow behaviour of powders is critical for consistent and efficient processing. The flow behaviour is affected by 1 ) the properties of the powder materials, e.g. particle size, shape, density, interactions between particles, etc. and 2) the processing parameters, which are related to a) processing equipment, e.g. geometry of hopper and outlet diameter, and b) processing conditions, e.g. feeder speed, environment.

Air pressure effects are very important particularly in the handling and dosing of fine and cohesive powders. For example, typical pharmaceutical formulations used in tablet production are relatively cohesive and poor flowing. Yet, it is possible to fill these powders into dies significantly smaller in diameter than the minimum orifice diameter trough which the powder would normally flow under ambient conditions. Clearly, there is an unseen mechanism that enables this operation. This mechanism was identified [1-3] and termed "suction fill" which is effected by the geometry and operating conditions present on rotary tablet presses. Suction fill involves powder-air interactions which were identified in the 1960 s but remained relatively understudied until recently. A detailed literature review that describes air-powder interactions in the context of powder flow is presented in Section 2.

This paper reports the experimental measurements of the mass flow rate of fine and cohesive powders under differential pressure conditions. The existing models are evaluated and a new dimensional model is proposed. This work is relevant for understanding powder handling and dosing processes involving fine and cohesive powders.

\section{Powder flow models}

Understanding the parameters affecting powder flow and discharge from hoppers and bins has been subject to considerable experimental and theoretical research. A comprehensive review of these models can be found in textbooks and monographs [4-7]. Traditional models developed for hopper discharge, including Beverloo [8], Brown and Richard's [6,9] minimum energy theorem, Davidson and Nedderman's [10] hour glass theory and Johansson's flow factor model [11] are summarised below. These classic models provide the basis for new developments incorporating air pressure effects, which are also detailed below.

Nedderman et al. [12] describes studies of mass flow rate of granular materials carried out by Hagen [13] as early as 1852. Deming and Mehring [14] investigated the effects of half angle of the hopper, orifice size, particle size and bulk density on the flow rate of powders. In 1952, Weighardt [15] proposed that the mass flow rate is a function of $(D-Z)^{2.5}$. This is similar to the well-known model introduced by Beverloo et al. [8] in 1961:

$$
\dot{m}_{B}=c \rho_{b} g^{0.5}(D-k d)^{2.5}
$$


where the value of the Beverloo constant, $c$, was found to be close to 0.58 and the term $k d$ ( $Z$ in Weighardt's relation) is a correction factor used to fit the experimental data to the model which is compatible with the concept of "empty annulus" proposed by Brown and Richards [6,9]. In Beverloo's study the mass flow rate was found to be independent of the height of the powder above the orifice.

The Beverloo model (Equation 1) is only applicable to cylindrical bunkers and core flowing hoppers. A pre-Beverloo correlation was developed for mass flow hoppers by Rose and Tanaka [16] where the mass flow rate was a function of the half angle of the hopper.

Using the "Minimum Energy Theorem", Brown and Richards $[6,17]$ developed a theoretical model to describe the flow rate of coarse and incompressible powders from bins and hoppers (Equation 2). This model was in good agreement with the experimental data obtained for coarse particles.

$$
\dot{m}=\frac{\pi}{6} \rho_{b} g^{0.5}(D-Z)^{2.5}\left(\frac{1-\cos ^{1.5} \alpha}{\sin ^{2.5} \alpha}\right)
$$

Davidson and Nedderman [10] used the approach known as "the Hour-Glass Theory" to determine the mass flow rate of incompressible coarse powders. The predicted flow rates using this model was found to be double the experimental values; and they concluded that this inconsistency was due to the effect of wall friction.

The models proposed for predicting the mass flow rate of bulk solids are often developed for noncohesive materials. Johanson [11] proposed a theoretical correlation for cohesive materials by including the effects of inertia in the equilibrium of a cohesive arch of uniform thickness:

$$
\dot{m}=\dot{m}_{B} \sqrt{\left(1-\frac{f f}{f f_{c}}\right)}
$$

In Equation 3, $\dot{m}_{B}$ is the mass flow rate of a non-cohesive material of the same density predicted using the Beverloo correlation, $f f$ is the flow factor and $f f_{c}$ is the critical flow factor at which arching occurs. This relation showed a good agreement for coarse materials however, larger experimental discrepancies were observed for fine powders.

The first major study of the effect of air pressure on the flow rate of bulk solids was carried out in 1964 by Bulsara et al. [18]. They applied additional air pressure on the top of the powder bed in the hopper and found that the mass flow rate is related to the differential pressure $(\Delta P)$ by

$$
\dot{m} \propto \Delta P^{0.5}
$$

McDougal and Knowles [19] modified this relation to the form of Equation 5 where $\Delta P_{0}$ is the adverse pressure gradient required to prevent flow. Resnick et al. [20] mentioned that in the absence of additional air pressure McDougal's relation should reduce to the expression for gravity flow and the mass flow rates should be closely related to the values predicted by the Beverloo correlation (Equation 1).

$$
\dot{m} \propto\left(\Delta P+\Delta P_{0}\right)^{0.5}
$$


The first theoretical work on air effects was carried out by Crewdson et al. [21] by including a modification to the acceleration of particles. In gravity flow, the particles accelerate because of their weight $\left(\rho_{b} g\right)$ while in the presence of an interstitial pressure gradient an additional body force will be applied. Based on this analogy the Beverloo correlation was modified for hoppers to:

$$
\dot{m}=\dot{m}_{B}\left(1+\frac{\Delta P}{\rho_{b} g r_{0}}\right)^{0.5}
$$

where $r_{0}$ is the radius of the free fall arch. This relation is similar to the model proposed by Bulsara [18]. Two modified versions of this model were presented for intermediate and high Reynolds numbers [7]. By assuming that there is no air percolation through the stagnant zone, Equation 6 can be used for flat-bottomed bins by treating it as a hopper of angle equal to that of a flowing core which can be assumed to be $45^{\circ}$ and $r_{0}=D / \sqrt{2}$ [19].

The mass flow rate predicted by the Beverloo correlation is significantly larger than the experimental values recorded for fine powders (with average particle size smaller than $400 \mu \mathrm{m}$ ) and it was suggested that the value of the coefficient should be modified. This effect was attributed by Nedderman [7] to the change of voidage and pressure gradient in powder bed. For coarse powders, the pressure gradient effects due to dilation are negligible but become significant for fine powders. The effects of negative pressure gradient was investigated experimentally by Verghese [22]. It was observed that the magnitude of pressure gradient at the vicinity of the orifice increased by decreasing particle size and a model for predicting mass flow rate was proposed

$$
\dot{m}=\dot{m}_{B}\left(1-\frac{\lambda}{\rho_{b} g d^{2}}\right)^{0.5}
$$

where $\lambda$ is a function of compressibility of the material and gas properties. They found that for a series of sands with small size distribution the mass flow rate can be predicted using $\lambda / \rho_{b} g=1.48 \times 10^{-8}$ $\mathrm{m}^{2}$. However, there is no reason why this value should be the same for other powders.

Donsi et al. [23] proposed a model to predict the mass flow rate of cohesive powders in aerated systems by modifying the relation developed by De Jang and Hoelen [24]. However, this model failed to capture the behaviour of poor flowing and cohesive powders.

$$
\dot{m}=0.55 \rho_{b}(1-\varepsilon)\left[\beta_{1}(Q / \varepsilon)^{2}+\beta_{2} Q / \varepsilon+g(D-1.5 d)^{5}\right]^{0.5}
$$

Barletta et al. [25] modified Donsi's relation assuming that cohesive powders flow in form of aggregates. They included the aggregate size and density in Donsi's relation which gave a better prediction, however, it still overestimated the mass flow rate. Wu et al. [26] and Schneider et al. [27] examined air pressure effects on powder discharge into closed cavities by conducting experiments using a linear shoe-die system in both air and vacuum. It was shown that the mass discharged into the die reduced by a factor of 2 for the experiments conducted in air.

In the past decades the flow of particulate materials has been modelled extensively using the Discrete Element Method (DEM) as described in textbooks and monographs (e.g. [28]) and the use of commercial and open source codes has become widespread. DEM allowed numerical experiments to study the effect of particle parameters on flow. For example, Li et al. [29] used DEM and obtained an expression for mass flow rate similar to Beverloo (Equation 1), and proposed that rather than being a 
constant, " $c$ " was dependent on the coefficient of friction between particles. Of particular importance for the subject of this paper are the methods that couple DEM with Computational Fluid Dynamics (CFD). These enabled further numerical experiments on the influence of air. For example, Guo et al. [30] examined die fill using a linear shoe/die system and arrived at an expression for mass flow rate that included the Archimedes number, which is a dimensionless group that includes particle size, solid and gas density and gas viscosity (Equation 9). Simulations of fluidisation or powder transfer have become routine; however, numerical studies have not yet delivered a mechanistic model of powder flow.

$$
\frac{\dot{m}}{\rho_{b} g^{1 / 2} l b_{0}{ }^{3 / 2}\left(1-k \frac{d}{b_{0}}\right)^{3 / 2}}=B\left(\operatorname{Ar} . \Phi_{p}\right)^{1 / 5}
$$

\section{Description and characterisation of powder materials using standard techniques}

Four grades of microcrystalline cellulose (MCC), Avicel PH101, PH102, PH200 and PH302 (manufactured by FMC BioPolymer), Calcium Phosphate (Anhydrous grade (known as A-Tab ${ }^{\circledR}$ ) manufactured by Innophos), labelled as ATAB in this paper; and Mannitol (grade Pearlitol 200SD manufactured by Roquette) were used in this study. These materials are pharmaceutical excipients used widely in tablet formulations. The choices of materials was made to represent powders with different average particle size, density, particle shape and cohesive behaviour.

A series of standard powder characterisations methods were used to measure bulk density, critical orifice diameter and the angle of repose of the materials using systems developed at the University of Leicester which are described elsewhere [31]. The properties of the materials are summarised in Table 1. The angle of repose quoted is for the system that has a base diameter of $75 \mathrm{~mm}$.

For convenience, $\mathrm{PH} 101, \mathrm{PH} 102$ and $\mathrm{PH} 302$ are described as "fine and cohesive" powders; and PH200, Mannitol and ATAB are referred to as "good" flowing powders. A formal classification based on angle of reposed was proposed by Carr [32].

Table 1. Properties of the six powders.

\begin{tabular}{|c|c|c|c|c|}
\hline Material & $\begin{array}{c}\text { Average Particle } \\
\text { Size, } \mu \mathrm{m}^{*}\end{array}$ & $\begin{array}{c}\text { Bulk Density, } \\
\mathrm{kg} / \mathrm{m}^{3 * *}\end{array}$ & $\begin{array}{c}\text { Critical Orifice } \\
\text { Diameter, } \mathrm{mm}^{* *}\end{array}$ & Angle of Repose, $^{* * *}$ \\
\hline PH101 & 50 & 0.309 & 30 & 45 \\
\hline PH102 & 100 & 0.318 & 26 & 40 \\
\hline PH302 & 100 & 0.428 & 24 & 45 \\
\hline PH200 & 180 & 0.363 & 11 & 34 \\
\hline ATAB & 180 & 0.725 & 3 & 36 \\
\hline Mannitol & 180 & 0.498 & 6 & 33 \\
\hline
\end{tabular}

* Provided by the manufacturer.

** Measured using the procedures described in reference [31].

Scanning Electron Microscope - SEM - (XL30 ESEM manufactured by Philips) images of the six powders are presented in Figure 1. The particle morphology of all four grades of MCC is similar [33]. 
Observations regarding the effect of average particle size are made for PH101, PH102 and PH200 which have similar bulk densities. The effect of bulk density is studied by comparing PH102 and PH302 which have similar average particle size.

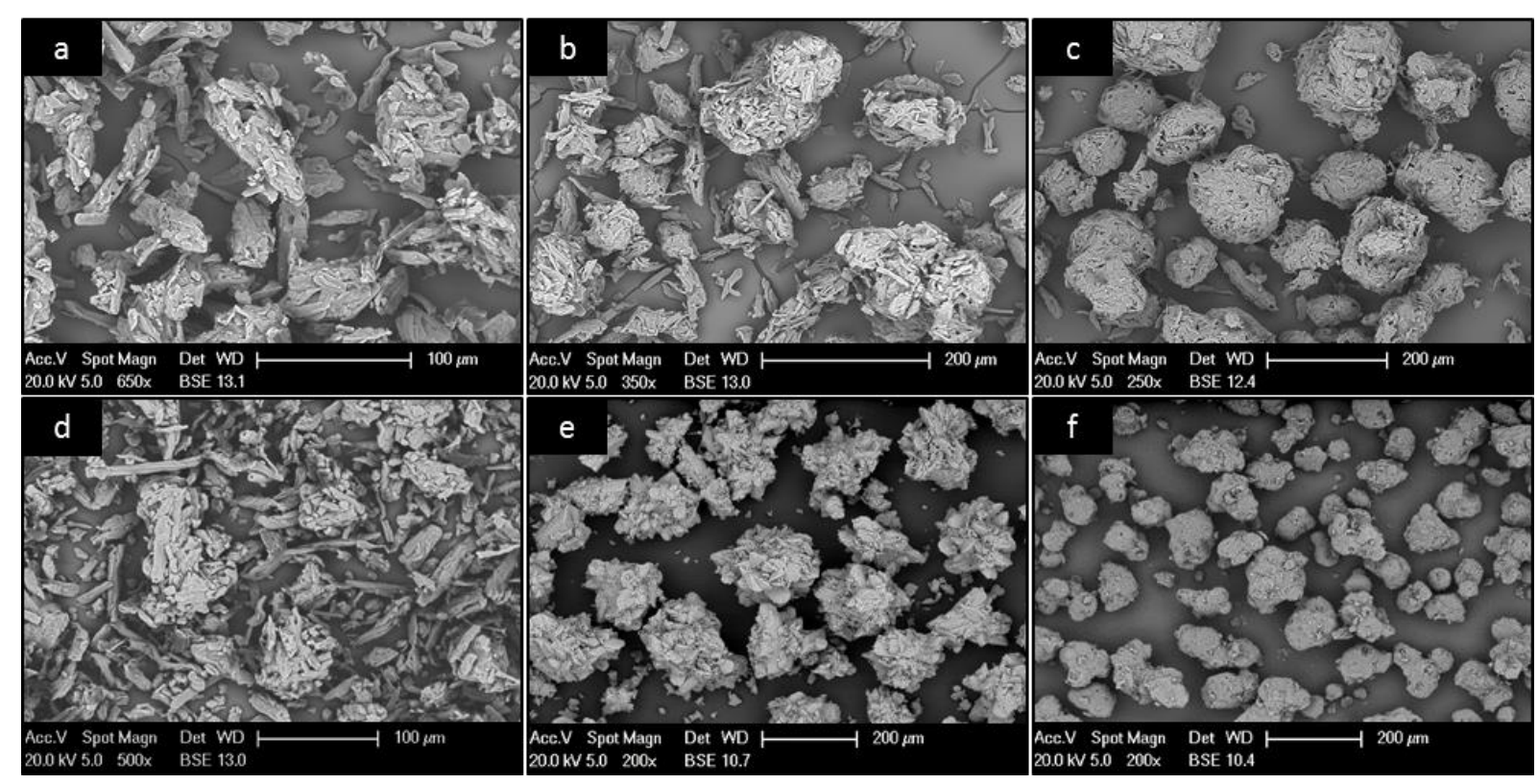

Figure 1. The SEM images of a) PH101, b) PH102, c) PH200, d) PH302, e) ATAB and f) Mannitol. Note: a-d are from reference [31].

Interactions between the interstitial gas and particles were characterised by measuring the permeability of the powders for a range of consolidation stresses using a FT4 Powder Rheometer [34]. These experiments were conducted using Nitrogen at $15{ }^{\circ} \mathrm{C}$ with fluid velocity set at $2 \mathrm{~mm} / \mathrm{s}$. Permeability values were determined using Darcy's Law [35] .

$$
Q=\frac{k A}{\mu} \frac{\Delta P}{L}
$$

The permeability measurement results are presented in Figure 2. The following powder ranking is obtained based on the permeability values from highest to lowest. This ranking remains the same for the different consolidation stresses applied part of the standard permeability measurement procedure as illustrated in Figure 2.

$$
\text { PH200 }=>\text { ATAB }=>\text { Mannitol }=>\text { PH102 }=>\text { PH101 }=>\text { PH302 }
$$




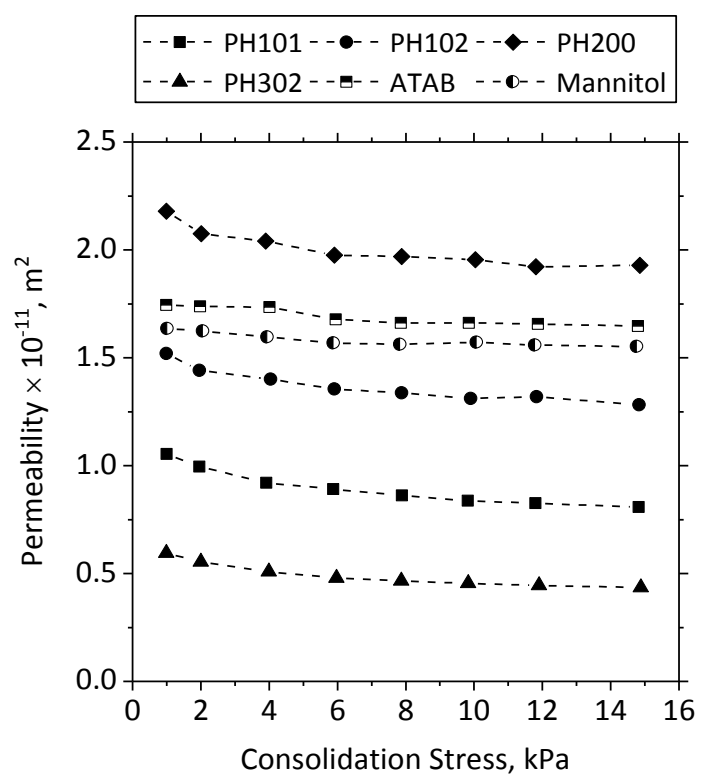

Figure 2. Permeability of the six powders determined at different consolidation stresses using a FT4 Powder Rheometer.

\section{Development and calibration of the powder flow characterisation system under differential pressure}

The experimental system consists of a critical orifice measurement device mounted above a vacuum chamber. The critical orifice measurement device is made up of a cylindrical section (diameter $68 \mathrm{~mm}$ and height $70 \mathrm{~mm}$ ) extended with a hopper at the top to accommodate a powder sample of approximately $1 \mathrm{~kg}$. The powder material is introduced through a conditioning device adapted from the Scott-Volumeter [36] and assembled above the hopper shown in Figure 3 for the purpose of ensuring consistent and repeatable initial conditions for the powder before testing. The tests reported in this paper are conducted using disks with orifice diameters of 5, 7 and $10 \mathrm{~mm}$, which are representative to die sizes used in pharmaceutical tablet pressing.

The powder is discharged from the critical orifice measurement device into a vacuum chamber underneath as illustrated in Figure 3. The differential pressure between the chamber and ambient atmospheric conditions is applied using a vacuum pump controlled by a precision vacuum regulator (Airtrol V-900-10 W/K).

The pressure inside the chamber is monitored using a differential pressure transducer (Sensirion SDP2000-L) and logged using a 16 bit resolution data acquisition device (National Instruments) driven by PC software (LabView). The calibration of the pressure transducer is validated against a calibrated manometer as illustrated in Figure 4. In the calibration system, the static pressure difference is generated using a Hot-wire Anemometer where the steady flow of air results in a pressure drop inside the unit (Figure 4) that can be adjusted using an air velocity control system. The output voltage of the differential pressure transducer is converted to pressure units using the manufacturer's relationship and compared with the manometer. The measurement errors are smaller than $2 \%$. 


\section{Experimental procedure}

A disk with a given orifice diameter is assembled into the system illustrate in Figure 3. A sample of 800 grams of powder is introduced into the hopper using the powder conditioning device. A prescribed differential pressure $(0-1400 \mathrm{~Pa})$ is applied in the vacuum chamber. The shutter below the orifice is opened using a release pin illustrated in Figure 3. The discharging powder is collected in a beaker placed on a scale inside the vacuum chamber. In order to measure the mass flow rate in steady state regime only, time measurement was started after 100 grams of powder was collected and the time taken for 600 grams of powder to flow through was recorded. During the flow process the top layers of the powder in the hopper was gently stirred by hand using an L-shaped rod to ensure the top surface of the bed was always covered with powder and to avoid "rathole" forming in the powder bed. Since powder flow is mostly influenced by the condition of the powder at the vicinity of the exit, agitation is believed to have a limited effect on powder flow and the differential pressure across the powder column was maintained during the flow rate measurement period. In the experiments where the powder discharged in open atmosphere (with no differential pressure applied) the lower cabinet is left open to avoid pressure increase in the chamber as the powder is introduced.

Flow rate measurements are taken under various differential pressures, which were increased in 200$300 \mathrm{~Pa}$ increments. A minimum of 12 measurements are taken for a given orifice diameter for each of the six materials.

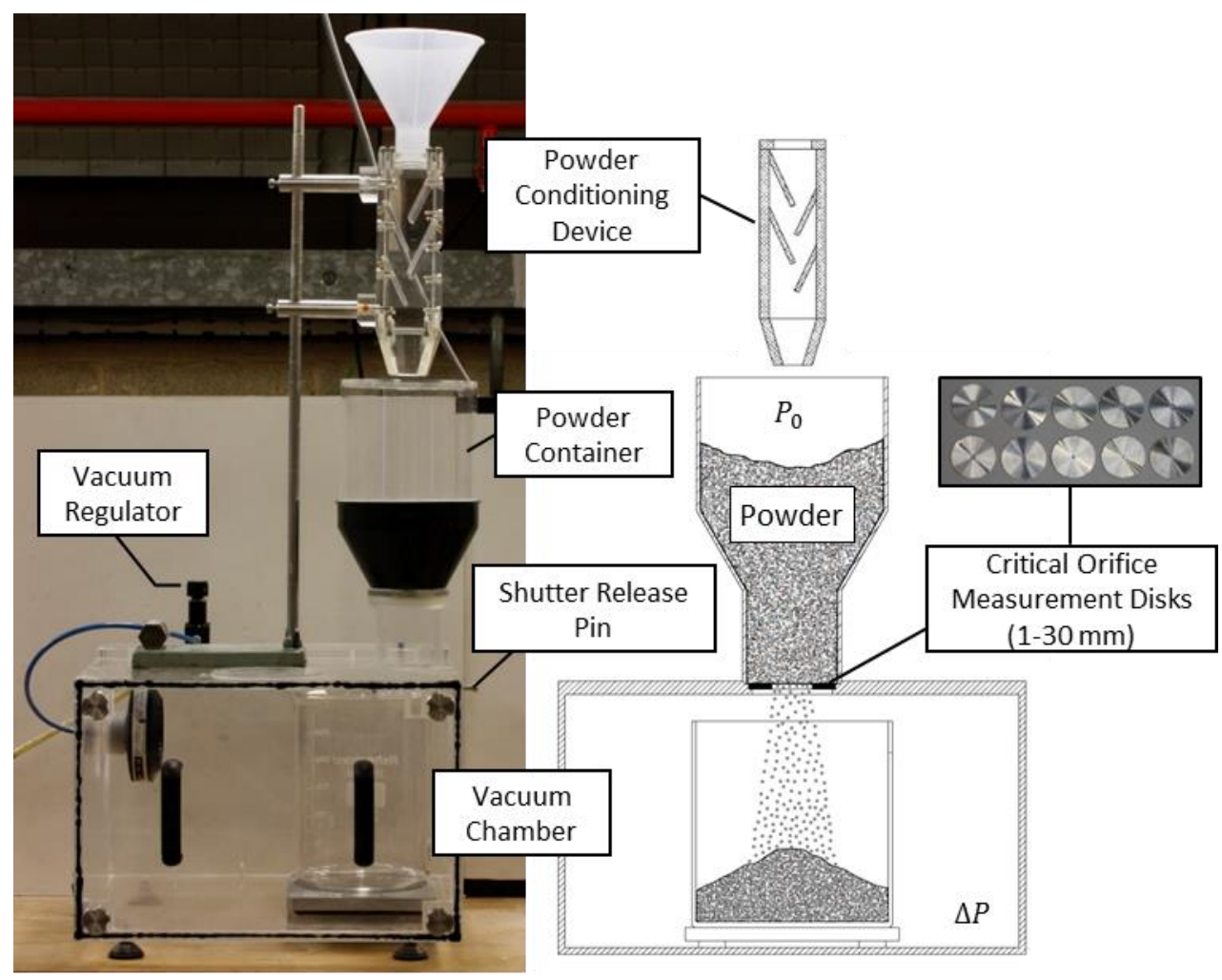

Figure 3. Device for measuring powder flow rate under differential pressure. 


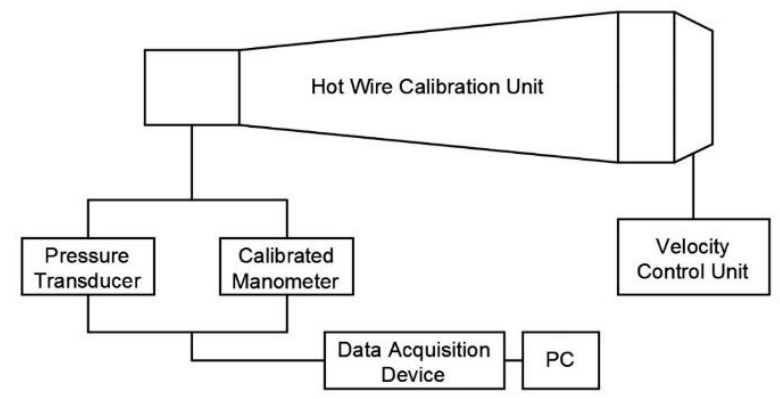

Figure 4. Schematic diagram of pressure transducer calibration procedure.

\section{Results and discussion}

\subsection{Observations of flow rate under differential pressure}

The term "differential pressure" refers to the pressure difference between the vacuum chamber and the ambient pressure averaged over the mass flow rate measurement period.

The mass flow rate of the four grades of microcrystalline cellulose measured under differential pressure is presented in Figure 5 for ready comparison of the flow behaviour of powders made of the same material but different particle size and density. In addition, the experimental measurements for all powders are presented in Figure 6. The following key observations are made:

- The flow rate increases when the exit diameter is increased

- The flow rate increases when the differential pressure is increased

- Under differential pressure conditions, the mass flow rate of "fine and cohesive" powders (PH101, PH102, PH302) can become higher than of "good" flowing powders (PH200, Mannitol, ATAB).

These observations are discussed in more detail below. Figure 5 presents the data for the four grades of MCC with the three regions corresponding to different orifice diameters, 5, 7, and $10 \mathrm{~mm}$ as indicated. It is evident that the mass flow rate increases when the orifice diameter and differential pressure are increased, as anticipated.

For experiments carried out in the ambient atmosphere (no differential pressure), the data point on the " $y$ " axis in Figure 6 suggest the following rank order of powders in terms of flowability starting from poor flow:

$$
\text { PH101 }=>\text { PH102 }=>\text { P302 }=>\text { PH200 }=>\text { Mannitol }=>\text { ATAB } .
$$

The exit diameters considered $(5,7$ and $10 \mathrm{~mm})$ are below the critical orifice diameter for $\mathrm{PH} 101$, PH102, PH302 and PH2O0 (for these materials the critical orifice diameters are 30, 26, 24 and $11 \mathrm{~mm}$ respectively). Therefore, the mass flow rate for these powders could not be measured in ambient atmosphere, hence the absence of data points for zero differential pressure in Figure 5. However, as the differential pressure was applied and increased above value necessary for flow initiation, the rank order of MCC powders is changed the new ranking starting from poor flow becomes: 


$$
\mathrm{PH} 200=>\text { PH102 }=>\text { PH101 }
$$

for larger differential pressures. The comparison above was limited to PH101, PH102 and PH200 because these powders have similar bulk density but different average particle size. This observation is valid for the three exit diameters in Figure 5. PH302, having a higher bulk density, exhibits higher mass flow rate than $\mathrm{PH} 102$ which has similar average size.

Figure 5 and 6 show that the mass flow rate of the fine and cohesive powders is comparable to that of the coarse powders under differential pressure (e.g. PH101 and PH200 on Figure 5). For the powders with higher critical orifice diameter higher differential pressure is required; and data is available above the flow initiation pressure only.

There are data points in Figure 5 and 6, which indicate that the poor flowing powders have higher mass flow rate than the good flowing powders; however, because of the scatter in the data and limited number of data points, a generalised statement cannot be made in this regard. Nevertheless it is interesting to note that mass flow rate of powders under differential pressure is relatively similar.

In terms of mass flow rate, ATAB and Mannitol outperform the other materials (Figure 6); however, it is important to note that their bulk density is also higher. For the exit diameter of $10 \mathrm{~mm}$ under high differential pressures, larger scatter is observed in the data. For larger orifice diameters, the powder flow period reduces significantly and the measurement errors increase .

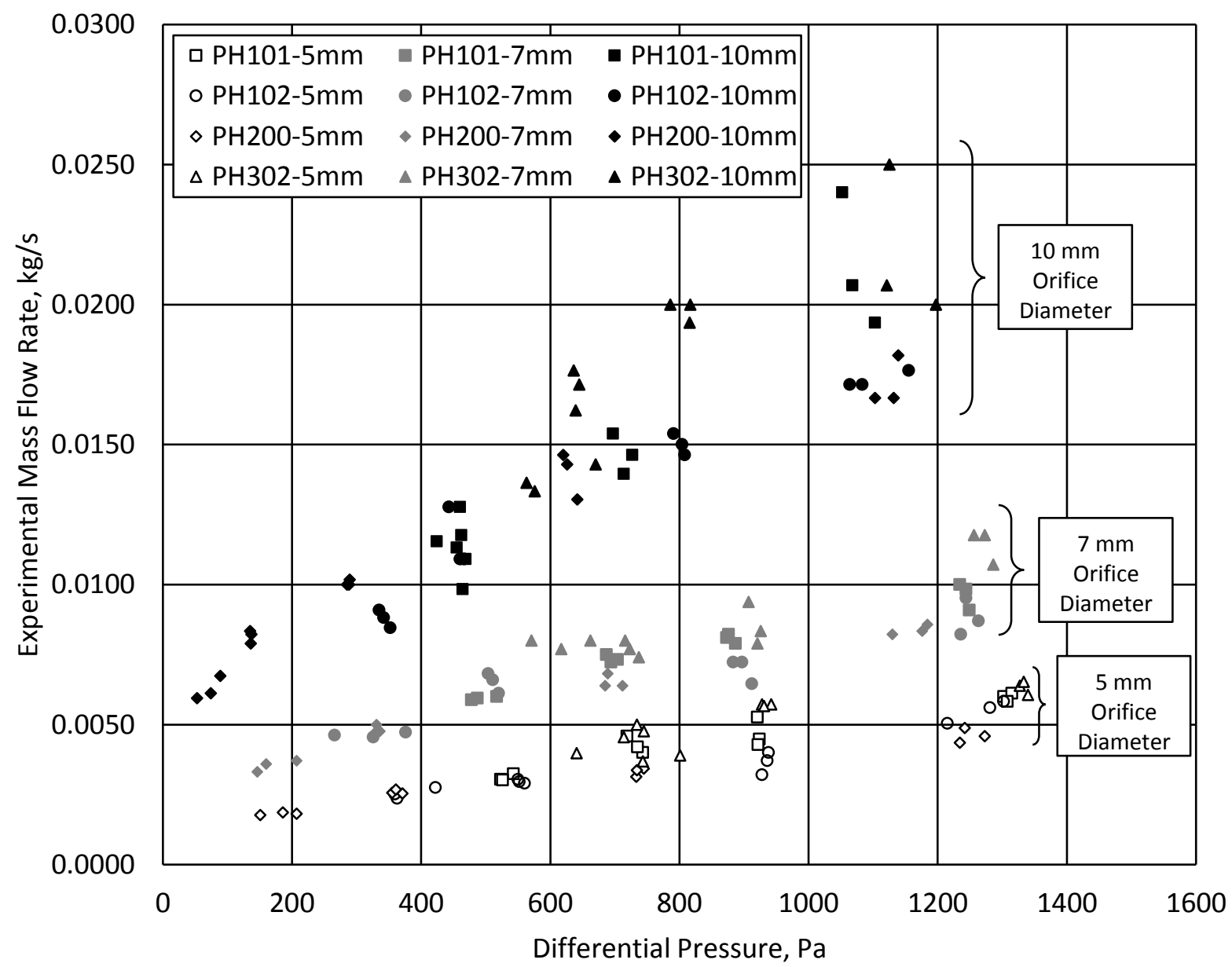

Figure 5. Experimental mass flow rate vs. differential pressure for four grades of MCC. 


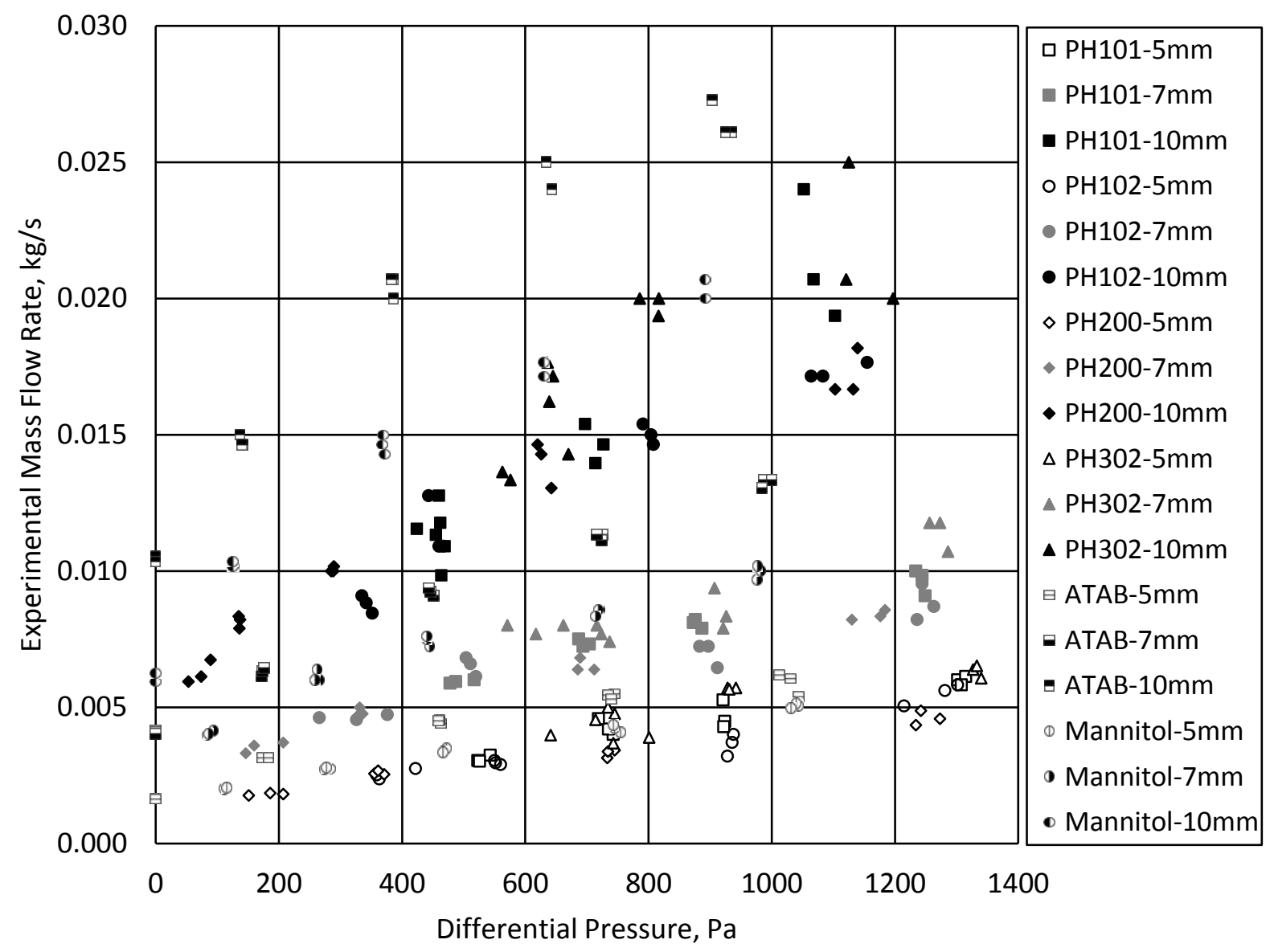

Figure 6. Mass flow rate vs. differential pressure measured for all powders.

In the next section, the existing powder flow models are employed to check their ability to describe the observed effects.

\subsection{Analysis of flow rate data using existing models}

The modified Crewdson model $[7,21]$ (Equation 11, obtained from Equation 6 with $r_{0}=D / \sqrt{2}$ ) was used to predict for the mass flow rate of the powders under the same conditions as used in the experiments and the results are presented in Figure 7.

$$
\dot{m}=\dot{m}_{B}\left(1+\frac{\sqrt{2} \Delta P}{\rho_{b} g D}\right)^{0.5}=c \rho_{b} g^{0.5}(D-k d)^{2.5}\left(1+\frac{\sqrt{2} \Delta P}{\rho_{b} g D}\right)^{0.5}
$$

For each data point the $\mathrm{x}$-coordinate represents the experimentally measured value of mass flow rate and the $y$-coordinate the value predicted by the Crewdson. Data points on the diagonal indicate perfect agreement between the experiment and model. The predicted values are above the diagonal indicating that the Crewdson model significantly over-predicts the flow rate for every test case.

The model of Crewdson [18] (established using $90-850 \mu \mathrm{m}$ diameter powders with interstitial pressure gradient) was developed considering that the interstitial gas pressure results in a change in acceleration of the particles and the term $\Delta P / \rho_{b} r_{0}$ was included into the Beverloo's correlation as an 
addition to the gravitational acceleration. Therefore, the exponent (0.5) cannot be changed for reasons of dimensional consistency.

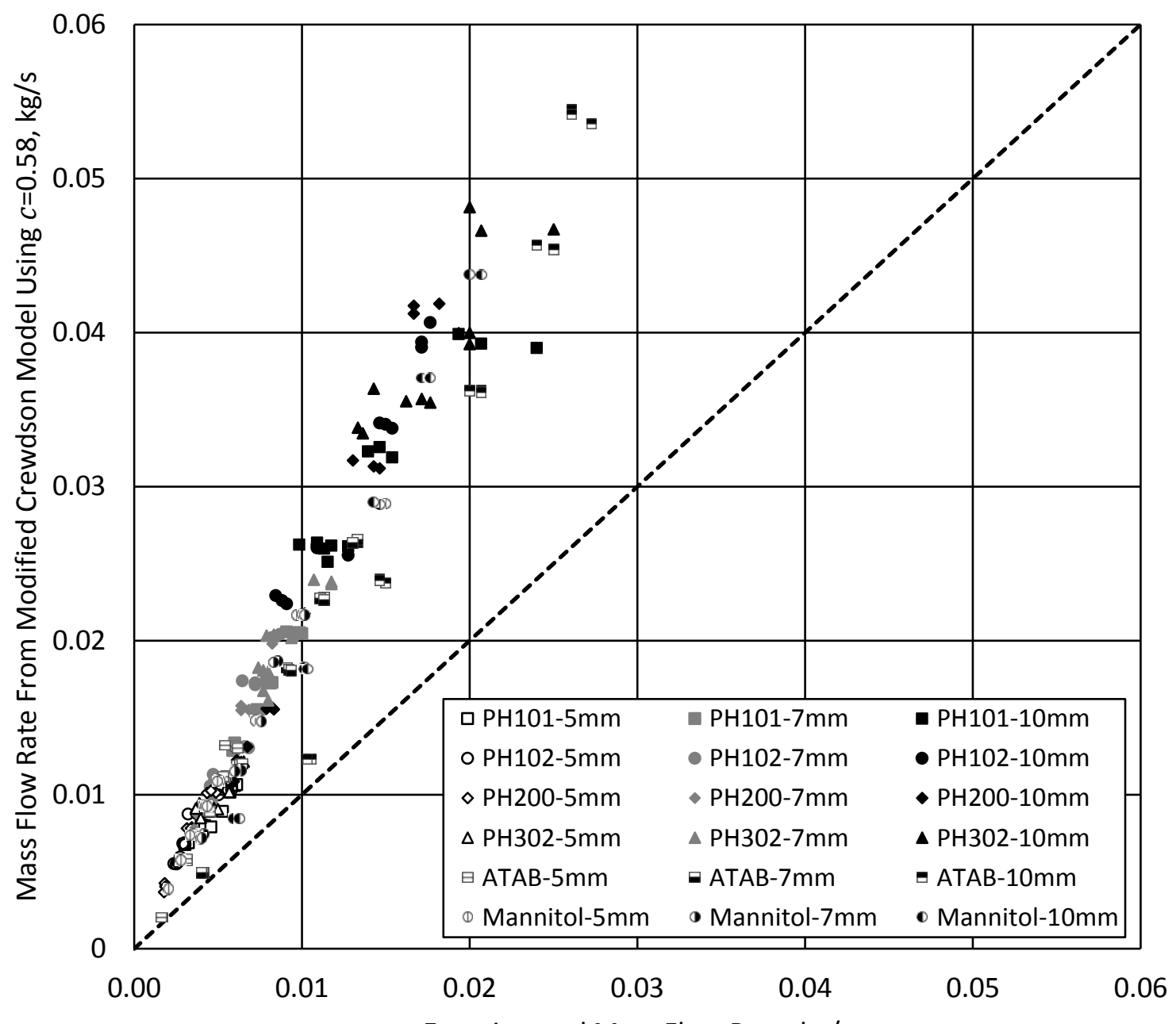

Figure 7. Mass flow rates calculated using Crewdson model with $c=0.58$ vs. mass flow rates measured experimentally for exit diameters of 5, 7 and $10 \mathrm{~mm}$.

It is noted that Equation 11 uses the value of 0.58 for the Beverloo constant. Nedderman [7] suggested that for fine powders the Beverloo constant ceases to be 0.58 and becomes strongly dependent of particle diameter. In the following we examined the possibility of considering $c$ (present in $m_{B}$ ) as a material dependent parameter. The procedure for obtaining $c$ for each material involves measuring the mass flow rate in ambient atmosphere (without applying differential pressure). Given the values of the critical orifice diameter (Table 1) this procedure could be applied only for ATAB and Mannitol as discussed in Section 6.1. For PH200 (critical orifice diameter $11 \mathrm{~mm}$ ) an extra set of experiments was carried out using a disk with an orifice diameter of $15 \mathrm{~mm}$ and the results are presented in Figure 8. The Beverloo coefficients determined for PH200, ATAB and Mannitol are indicated in Table 2. 
Table 2. Modified Beverloo coefficient determined experimentally for "good" flowing materials

\begin{tabular}{|c|c|}
\hline Material & Modified Beverloo coefficient \\
\hline PH200 & 0.42 \\
\hline ATAB & 0.48 \\
\hline Mannitol & 0.42 \\
\hline
\end{tabular}

PH101, PH102 and PH302 have large critical orifice diameter (Table 1). Their flow rate in air could not be measured using the current system because the flow through a large orifice occurs at a high rate for which the scale described in Section 4 was not sufficiently sensitive. Therefore, the adjusted Beverloo constants were not determined experimentally for these powders.

Comparison of the results obtained from the original and modified Crewdson model and the experimental results showed that the existing models significantly over predict the mass flow rates observed. This is demonstrated on Figure 8 for PH2O0 as a reference. A new model is proposed in the following section.

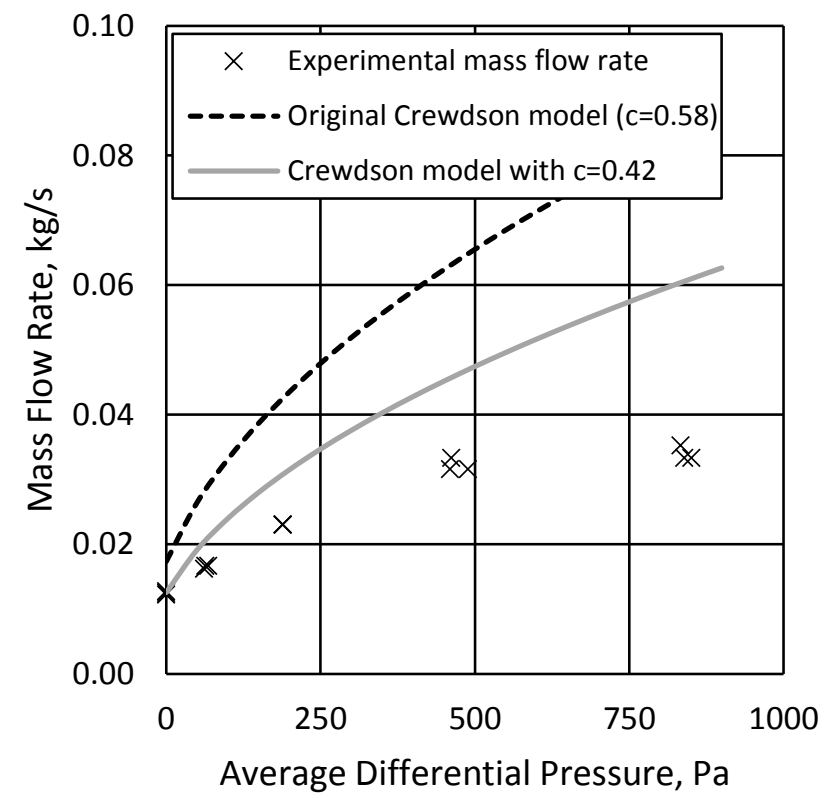

Figure 8. Mass flow rate for $\mathrm{PH} 200$ through orifice diameter of $15 \mathrm{~mm}$ indicating measurement and prediction using the Crewdson model with $c=0.58$ (original model) and $c=0.42$ (modified model).

\subsection{Dimensional model development and validation}

The Beverloo model (Equation 1) was developed for flow in ambient atmosphere. To account for the effect of differential pressure Crewdson et al. and Nedderman [7,21] proposed a modification whereby an additional acceleration term was added to gravity. However, as described above, Nedderman [7] concluded that $c$ must be a function of particle size. As shown in Figure 8 , this approach does not describe the behaviour of common pharmaceutical excipients. In fact in Equation 11, $c$ is the only parameter that could be immediately adjusted for a given powder, or considered a material dependent parameter.

In order to follow scientific rigour and consistency with existing models and reasons of practical use the dimensional model proposed below was guided by the following principles: 
1) follow the Buckingham $\Pi$ theorems [37];

2) reduce to Beverloo model in absence of differential pressure; and

3) contain material parameters that can be calibrated using simple tests using the methods presented.

We propose to define the total mass flow rate $(\dot{m})$ as the sum of the modified Beverloo correlation $\left(\dot{m}_{B}\right)$ and a term resulted from the differential pressure applied $\left(\dot{m}_{p}\right)$.

$$
\dot{m}=\dot{m}_{B}+\dot{m}
$$

The parameters involved in powder flow process under differential pressure can be listed as:

- mass flow rate induced by differential pressure $\left(\dot{m}_{P}\right)$;

- differential pressure $(\Delta P)$;

- bulk density of powder $\left(\rho_{b}\right)$;

- acceleration due to gravity $(g)$;

- $\quad$ particle size $(d)$; and

- orifice diameter $(D)$.

There are also other parameters like viscosity of air $(\mu)$, coefficient of friction between particles $\left(\mu_{p}\right)$ and between particles and the wall $\left(\mu_{p w}\right)$, shape of the particles and adhesion between the particles that contribute. Thus $\dot{m}_{P}$ can be written as a function of $\Delta P, \rho_{b}, g, d, D, \mu, \mu_{p}, \mu_{p w}$, particle shape and adhesion i.e.

$$
\dot{m}_{P}=f\left(\Delta P, \rho_{b}, g, d,, D, \mu, \mu_{p}, \mu_{p w}, \text { shape, adhesion }\right)
$$

Dimensional analysis and the Buckingham $\Pi$ theorem [37] is applied to develop a model to predict the mass flow rate of powders as a function of the differential pressure applied. In the analysis, the parameters $\rho_{b}, g$ and $D$ were taken as the independent variables. Consequently, the $\pi$ groups of $\overline{m_{P}}$ and can be formed as

and

$$
\overline{\dot{m}_{P}}=\frac{\dot{m}_{P}}{\rho_{b} g^{0.5} D^{2.5}}
$$

$$
\overline{\Delta P}=\frac{\Delta P}{\rho_{b} g D}
$$

Therefore

$$
\overline{\dot{m}_{P}}=f\left(\overline{\Delta P}, \rho_{b}, g, d, D, \mu, \mu_{p}, \mu_{p w}, \text { shape, adhesion }\right)
$$

The experimental data presented in Figure 6 could be fitted with a power law relation. We write in dimensionless form

or

$$
\overline{\dot{m}_{p}}=a(\overline{\Delta P})^{n}
$$

$$
\frac{\dot{m}_{P}}{\rho_{b} g^{0.5} D^{2.5}}=a\left(\frac{\Delta P}{\rho_{b} g D}\right)^{n}
$$


where $a$ and $n$ are material dependent parameters. Since the mass flow rate of powders is determined exclusively by the condition of the powder in the vicinity of the exit, $\Delta P / H$ is a more appropriate representation of the pressure gradient near the orifice. The height of powder bed can be implemented into $\overline{\Delta P}$ as an additional $\pi$ group.

$$
\bar{H}=\frac{D}{H}
$$

The local pressure gradient is also influenced by the restricted gas cross section due to the orifice i.e. $\Delta P \approx A_{c} / A_{o}$. Including these effects into Equation 18 we have

or

$$
\frac{\dot{m}_{P}}{\rho_{b} g^{0.5} D^{2.5}}=a\left(\frac{\Delta P}{\rho_{b} g D} \times \frac{D}{H} \times \frac{A_{c}}{A_{o}}\right)^{n}
$$

$$
\frac{\dot{m}_{P}}{\rho_{b} g^{0.5} D^{2.5}}=a\left(\frac{\Delta P D_{c}^{2}}{\rho_{b} g H D^{2}}\right)^{n}
$$

Substituting Equations 1 (without the inclusion of the empty annulus effect) and 21 into Equation 12 we have

$$
\dot{m}=c \rho_{b} g^{0.5} D^{2.5}+a \rho_{b} g^{0.5} D^{2.5}\left(\frac{\Delta P D_{c}^{2}}{\rho_{b} g H D^{2}}\right)^{n}
$$

or

$$
\frac{\dot{m}}{\rho_{b} g^{0.5} D^{2.5}}=c+a\left(\frac{\Delta P D_{c}^{2}}{\rho_{b} g H D^{2}}\right)^{n}
$$

where $c$ is the Beverloo coefficient adjusted for the material to give a correct prediction of the mass flow rate under no differential pressure, consistent with the condition identified by Nedderman [7].

To account for the empty annulus effect [6] the correction factor $k d$ is introduced where $k$ is a constant based on the shape of the particles. The proposed model is:

$$
\frac{\dot{m}}{\rho_{b} g^{0.5}(D-k d)^{2.5}}=c+a\left(\frac{\Delta P D_{c}{ }^{2}}{\rho_{b} g H D^{2}}\right)^{n}
$$

The parameters that are not explicitly appearing in the model are in fact included in the empirical parameters $c, a$ and $n$.

Using the values of the adjusted Beverloo coefficient $c$ (Table 2) for PH200, ATAB and Mannitol, the experimental data was fitted using Equation 24 and the values of material dependent parameters $a$ and $n$, presented in Table 3, were obtained. In the fitting, the particles were considered spherical $(k=1.5)$. 
Table 3. Flow parameters for the "good" flowing materials.

\begin{tabular}{|c|c|c|c|c|}
\hline Material & $c$ & $a$ & $n$ & $R^{2}$ \\
\hline PH200 & 0.42 & 0.077 & 0.49 & 0.95 \\
\hline ATAB & 0.48 & 0.089 & 0.45 & 0.94 \\
\hline Mannitol & 0.42 & 0.084 & 0.48 & 0.94 \\
\hline
\end{tabular}

For PH101, PH102 and PH302 the Beverloo coefficient could not be measured (Section 5.2). Considering that the Beverloo coefficient is strongly dependent of particle size [7], the value of c must be smaller for fine powders. However, fitting the experimental data of PH101, PH102 and PH302 using Equation 24 leads to the $c$ values of $0.90,1.00$ and 1.00 respectively. These are significantly larger than the values obtained for coarse powders (Table 3 ) and even larger than the value proposed by Beverloo (0.58) for free flowing materials. Therefore, it can be concluded that $c$ should be determined experimentally before fitting $a$ and $n$ to the data.

However, in absence of flow rate measurements in ambient air for PH101, PH102 and PH302 the following procedure was used to estimate values of $c$ for these materials. When fitting the values for $a$ and $n$ for the coarse powders it was observed that equally good fits (in terms of the coefficient of determination) could be obtained by setting a constant value $n=0.5$. Additionally, by considering $n=0.5$, the model will become similar to observations made by Bulsara [18] (Equation 4) on the relation between discharge rate and differential pressure. Therefore, it is assumed that $n=0.5$ for the other three MCC materials (PH101, PH102 and PH302) as well. Having fixed $n$, the values of $c$ and $a$ are determined from fitting the experimental data with Equation 24 which are presented in Table 4. For PH200, ATAB and Mannitol, the values of $a$ determined using the measured $c$ and fixed $n=0.5$ are also presented in Table 4.

Table 4. The values of the coefficients used to predict the mass flow rate.

\begin{tabular}{|c|c|c|c|c|}
\hline Material & $c$ & $a$ & $n$ & $R^{2}$ \\
\hline PH101 & 0.32 & 0.099 & 0.5 & 0.88 \\
\hline PH102 & 0.32 & 0.086 & 0.5 & 0.85 \\
\hline PH302 & 0.40 & 0.085 & 0.5 & 0.85 \\
\hline PH200 & 0.42 & 0.074 & 0.5 & 0.95 \\
\hline ATAB & 0.48 & 0.070 & 0.5 & 0.93 \\
\hline Mannitol & 0.42 & 0.073 & 0.5 & 0.94 \\
\hline
\end{tabular}

Using the parameters in Table 4 the mass flow rate predicted by Equation 24 for all materials is compared with the experimental measurements as presented in Figure 9. It is evident that the proposed model (Equation 24) is valid. It can be concluded that the proposed model is able to describe powder flow under differential pressure adequately. Large deviations are observed for high mass flow rates. However, these measurements also present larger experimental errors and scatter. 


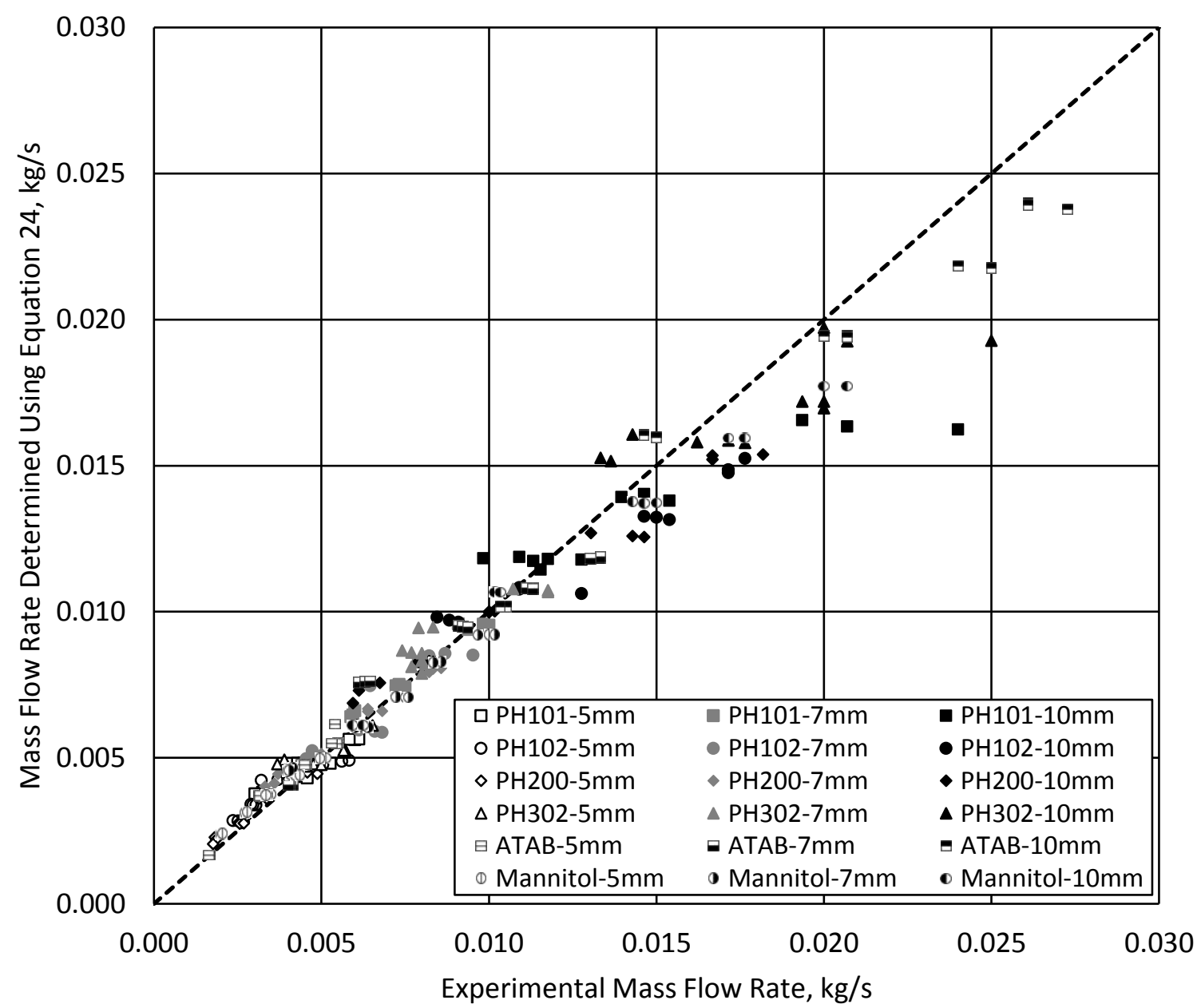

Figure 9. Mass flow rates calculated from the dimensional model vs experimental mass flow rates for all powders using exit diameters 5,7 and $10 \mathrm{~mm}$.

\section{Concluding remarks}

Standard experimental procedures such as angle of repose and critical orifice diameters can be used to rank powders in terms of flowability. However, the rank order of powders characterised by different techniques may be inconsistent. Conventional flow characterisation techniques were shown to have limitations for flow regimes where air-powder interactions are significant.

Orifice flow experiments under differential pressure show that the flow rate increases when the exit diameter is increased, and when the differential pressure is increased. Depending on the magnitude of differential pressure applied, rank order of powders in terms of flowability evolves and at higher differential pressures the mass flow rate of fine and cohesive powders can become higher than of good flowing powders.

The mass flow rate of fine and cohesive powders was measured experimentally. Existing models, such as Crewdson's (Equation 11), which was developed for coarse free flowing sands under differential pressure were found inadequate to describe the flow behaviour of fine and cohesive powders under differential pressure which represent an important class of powders used in pharmaceuticals, food, fine chemicals and other industries. 
Crewdson's model was developed for the flow of coarse free flowing sands under differential pressure [20]. However, this model does not describe adequately the flow of fine and cohesive powders. Dimensional analysis was used to provide a basis for constructing a new relationship, Equation 24, to predict mass flow rate under differential pressure.

The proposed relationship (Equation 24) includes three material parameters:

- $\quad c$ is a proportionality constant for the flow of powder in ambient atmosphere. The values listed in Table 4 are generally smaller for "poor" flowing powders and increase for "good" flowing powders classed according to the conventional flowability measures in Table 1.

- $a$ is a proportionality constant in the terms that includes the differential pressure, which offers increased flexibility to the model for use with a wider range of powders.

- $n$ is an exponent to account for the effect of differential pressure and it was considered as a constant value $n=0.5$. This makes the model consistent with the observations made by Bulsara [18] regarding the relation between the mass flow rate and differential pressure.

The differential pressure in Crewdon's model (Equation 11) affects all powder materials in the same way. However, it was shown in Section 5.1 that the differential pressure influences the flowability of powders differently: in simple terms the poor flowing powders "benefit more" than good flowing powders. The proposed model (Equation 24) has a general form suitable to discern this effect, particularly because $a$ is an adjustable material parameter which ensures good reproduction of the trends observed experimentally.

Equation 24 reduces to a Beverloo type relation provided that $c$ is determined experimentally from flow measurement in ambient atmosphere which is consistent with the studies described earlier $[19,20]$. For exit diameters smaller than the critical orifice diameter the mass flow rate in ambient atmosphere will be zero. To calibrate $a$ it is necessary to perform flow rate measurements under differential pressure.

The calibrated model can be used for practical applications where the exit diameter can be larger as well as smaller than the critical orifice diameter. The latter case is of significant practical importance. For example, many pharmaceutical formulations are poor flowing due to the cohesive nature of typical active pharmaceutical ingredients, e.g. the critical orifice diameter of active ingredients can be larger than $20 \mathrm{~mm}$ [38]. Pharmaceutical tablets are made by compressing the powder in dies of typical diameters under $10 \mathrm{~mm}$. Die filling is made possible [1,2] by the suction fill effect, whereby the downward movement of the lower punch creates a low pressure environment during die fill. Typical tablet presses produce 0.5-1 million tablets per hour [2]; such productivity is enabled by the enhanced mass flow rate into the die under differential pressure. The proposed model can be applied for powder handling and dosing processes enabled by differential pressure.

Powder flow can be viewed at particle level or bulk powder. At particle level the flow phenomenon is affected by a large number of particle characteristics such as particle size and size distribution, particle shape, morphology, friction and adhesion between particles, etc. Relevant properties at bulk level include cohesion and internal friction angle, which can be measured using shear testing methods. The model has empirical parameters ( $c$ and $a$ ) which can be determined from simple experiments as described above. While parameter $c$ can be related to bulk or particle properties [7,29], parameter $a$ requires flow measurement under differential pressure. 
The flow of powders in ambient atmosphere is complex due to interactions between air and powder. Whilst particle or bulk material properties can be measured and data is available, the parameters do not cover the experimental space for explicit inclusion in dimensional groups. However, the model proposed can be of significant practical use, bridge the gap between observations, and guide future work on models and mechanisms for powder flow.

Due to the complex nature of powder flow and the multitude of interrelated material and process parameters, the development of a mechanistic model is not yet possible. A small magnitude of differential pressure (e.g. a few hundred $\mathrm{Pa}$ ) reduces the critical diameter and increases the flow rate significantly for all powders tested. This effect can be utilised advantageously in a number of practical powder handling and dosing operations. The proposed model for powder flowability (Equation 24) was validates for fine and cohesive powders and can therefore be of significant use for formulation and process design.

\section{Acknowledgement}

Thanks to FMC Biopolymer for providing the four grades of microcrystalline cellulose and AstraZeneca for Mannitol and calcium phosphate powders.

\section{References}

[1] I.C. Sinka, A.C.F. Cocks, Evaluating the flow behaviour of powders for die fill performance, Powder Metallurgy. 52 (2009) 8-11.

[2] S. Jackson, I.C. Sinka, A.C.F. Cocks, The effect of suction during die fill on a rotary tablet press, European Journal of Pharmaceutics and Biopharmaceutics. 65 (2007) 253-256.

[3] L.A. Mills, I.C. Sinka, Effect of particle size and density on the die fill of powders, European Journal of Pharmaceutics and Biopharmaceutics. 84 (2013) 642-652.

[4] A.W. Jenike, Storage and flow of solids, Bulletin No. 123 of the Utah engineering experiment station, University of Utah. 53 (1964).

[5] D.M. Walker, An approximate theory for pressures and arching in hoppers, Chemical Engineering Science. 21 (1966) 975-997.

[6] R.L. Brown, J.C. Richards, Principles of Powder Mechanics, Pergamon Press, United Kingdom, 1970.

[7] R.M. Nedderman, Statics and Kinematics of Granular Materials, Cambridge University Press, United Kingdom, 1992.

[8] W.A. Beverloo, H.A. Leniger, J. van de Velde, The flow of granular solids through orifices, Chemical Engineering Science. 15 (1961) 260-269.

[9] R.L. Brown, Profile of Flow of Granules through Apertures, Trans.Inst Chem.Engrs. 38 (1960) 243256. 
[10] J.F. Davidson, R.M. Nedderman, The hour-glass theory of hopper flow, Trans.Inst.Chem.Eng. 51 (1973) 29-35.

[11] J.R. Johanson, A rolling theory for granular solids, Journal of Applied Mechanics. 32 (1965) 842848.

[12] R.M. Nedderman, U. Tüzün, S.B. Savage, G.T. Houlsby, The flow of granular materials-I: Discharge rates from hoppers, Chemical Engineering Science. 37 (1982) 1597-1609.

[13] G. Hagen, Berichtuber die zur Bekanntmachung geeigneten Verhandlungen der K oniglich Preussischen, 35 (1852) 35-42.

[14] W.E. Deming, A.L. Mehring, The Gravitational Flow of Fertilizers and Other Comminuted Solids, Ind. Eng. Chem. 21 (1929) 661-665.

[15] k. Weighardt, Studies on the flow of sand, Ingenieur-Archiv. 20 (1952) 109.

[16] H.E. Rose, T. Tanaka, Rate of discharge of granular materials from bins and hoppers, The Engineer. 208 (1959) 465-469.

[17] R.L. Brown, Minimum energy theorem for flow of dry granules through apertures, Nature. 191 (1961) 458-461.

[18] P.U. Bulsara, F.A. Zenz, R.A. Eckert, Pressure and additive effects on flow of bulk solids, Industrial \& Engineering Chemistry Process Design and Development. 3 (1964) 348-355.

[19] I.R. McDougal, G.H. Knowles, Flow of particles through orifices\&nbsp; Transactions of the Institution of Chemical Engineers and the Chemical Engineer. 47 (1969) T79.

[20] W. Resnick, Y. Heled, A. Klein, E. Palm, Effect of differential pressure on flow of granular solids through orifices, Industrial \& Engineering Chemistry Fundamentals. 5 (1966) 392-396.

[21] B.J. Crewdson, A.L. Ormond, R.M. Nedderman, Air-impeded discharge of fine particles from a hopper, Powder Technol. 16 (1977) 197-207.

[22] T.M. Verghese, The discharge of fine powders from conical hoppers, Chemical Engineering Science. 50 (1995) 3143-3153.

[23] G. Donsi, G. Ferrari, M. Poletto, P. Russo, Gas pressure measurements inside an aerated hopper, Chem. Eng. Res. Design. 82 (2004) 72-84.

[24] J. De Jong, Q. Hoelen, Cocurrent gas and particle flow during pneumatic discharge from a bunker through an orifice, Powder Technol. 12 (1975) 201-208.

[25] D. Barletta, G. Donsi, G. Ferrari, M. Poletto, P. Russo, Solid flow rate prediction in silo discharge of aerated cohesive powders, AIChE J. 53 (2007) 2240-2253.

[26] C.-. Wu, A.C.F. Cocks, Flow behaviour of powders during die filling, Powder Metallurgy. 47 (2004) 127-136. 
[27] L. Schneider, I.C. Sinka, A. Cocks, Characterisation of the flow behaviour of pharmaceutical powders using a model die-shoe filling system, Powder Technol. 173 (2007) 59-71.

[28] F. Radjai, F. Dubois, Discrete-Element Modeling of Granular Materials, Wiley, United States, 2011.

[29] T. Li, H. Zhang, M. Liu, Z. Huang, H. Bo, Y. Dong, DEM study of granular discharge rate through a vertical pipe with a bend outlet in small absorber sphere system, Nucl. Eng. Des. 314 (2017) 1-10.

[30] Y. Guo, K.D. Kafui, C. Wu, C. Thornton, J.P. Seville, A coupled DEM/CFD analysis of the effect of air on powder flow during die filling, AIChE J. 55 (2009) 49-62.

[31] R. Baserinia, I.C. Sinka, P. Rajniak, Vacuum assisted flow initiation in arching powders, Powder Technol. 301 (2016) 493-502.

[32] R.L. Carr, Evaluating flow properties of solids, Chem.Eng. 72 (1965) 163-168.

[33] A. Katdare, M. Chaubal, Excipient Development for Pharmaceutical, Biotechnology, and Drug Delivery Systems, CRC Press, United States, 2006.

[34] R. Freeman, Measuring the flow properties of consolidated, conditioned and aerated powdersa comparative study using a powder rheometer and a rotational shear cell, Powder Technol. 174 (2007) 25-33.

[35] H. Darcy, Les Fontaines Publiques De La Ville De Dijon: Exposition Et Application..., Victor Dalmont, France, 1856.

[36] United States Pharmacopeial Convention, USP29-NF24, 2006: U. S. Pharmacopeia [and] National Formulary, United States Pharmacopeial, United States, 2006.

[37] J.C. Gibbings, Dimensional Analysis, Springer Science \& Business Media, United States, 2011.

[38] L. Cai, L. Farber, D. Zhang, F. Li, J. Farabaugh, A new methodology for high drug loading wet granulation formulation development, Int. J. Pharm. 441 (2013) 790-800. 